\title{
ISOTHERMAL EXTRUSION OF NON-DILUTE FIBER SUSPENSIONS
}

\author{
TASOS C. PAPANASTASIOU and A.N. ALEXANDROU
}

Department of Chemical Engineering and Department of Mechanical Engineering, The University of Michigan, Ann Arbor, Michigan 48109 (U.S.A.)

(Received May 23, 1986; in revised form February 4, 1987)

\section{Summary}

The extrusion of a rod-like fiber suspension is a Newtonian solvent, as a first step to the fast and inexpensive production of composite materials, is investigated. The analysis is carried out by means of an integral constitutive equation for a non-dilute suspension, streamlined finite element for liquid with memory, and Newton iteration of nonlinear integro-differential equations. The predictions show substantial differences between dilute and nondilute fiber suspension regarding the processing conditions (pressure drop, velocity distribution, die-swell) and the resulting fiber orientation. Nondilute fiber suspensions exhibit substantial shear-thinning and negligible elasticity as evidenced by the small die-swell, and fiber concentration viscosity-thickening as evidenced by the large pressure drop. The fiber orientation is computed by solving the orientation distribution function along selected streamlines of the complex velocity field. It is shown that the fiber orientation far downstream can be made independent of the random fiber orientation at the inlet.

\section{Introduction}

For years, a major effort of the aerospace industry, in an effort to increase the speed and efficiency of aircraft, has been to build lighter, yet stronger aircraft. This trend has led to the development and use of a class of materials which have high strength-to-weight ratios. These materials consist primarily of continuous graphite fibers and epoxy resins. Both the cost of the fibers and resins are very high. Even more prohibitory to their use in commercial applications are the long processing and curing times required in the batch operations involved. Thus, the materials employed by automobile, 
and other consumer products, manufacturers more often include glass fibers which are usually short, and rapid-cure polyester resins. The macroscopic mechanical properties of these composites-strength and toughness, or elongation to failure-depend on the orientation of the fiber in the flow field at the time of solidification or curing which, of course, prevails in the solid state. Thus, it is extremely important to develop theories and methods of analysis for understanding complicated flow patterns and implied fiber orientations.

Despite their major importance in defining the final mechanical properties of the composite, processing operations have been the subject of little theoretical research effort and investigation mainly for two reasons: (a) the liquids involved during these operations are rheologically complex, exhibiting strong nonlinear viscoelastic behavior, and the flow fields are further complicated by the presence of the fibers; and (b) research into composite materials is primarily focused on the determination of the properties of the resulting solid part which are directly related to the applications of these materials.

Past methods for determining the fiber orientation have been based on experimental techniques such as the use of $X$-rays [1] and contact micro-radiography techniques [2]. These measurements can only be performed on the finished molded specimens and so their value is more diagnostic than process controlling. Recent efforts have been focused towards the predictive determination of the fiber orientation state from the flow kinematics $[3,4]$. In general, the motion of particles in a viscous medium has been a continuing topic of interest in fluid mechanics. A comprehensive review of this area has been provided by Leal [5]. The finite-element method was introduced quite successfully very recently to study flow orientation in relatively simple flows of dilute fiber suspensions in Newtonian and non-Newtonian media [6,7]. The predicted orientation patterns were similar to those observed in experimental studies by Goettler [8]. An excellent review of suspensions in Newtonian and non-Newtonian solvents has been given very recently by Metzner [9].

The preceding analyses apply only to cases of isolated particles in simple flow fields and do not take in account interactions among particles or fibers which were found to give rise to significant viscoelastic phenomena in semiconcentrated suspensions. These phenomena include transient behavior [10], yields stresses [11,12], shear thinning [13,14], and volume fraction dependence [15], in pure shearing flows. Similarly, in elongational flows, increased elongational viscosities have been reported by several investigators [16-18] even at very low concentrations [19]. In an attempt to predict these rheological data, Dinh and Armstrong [20] developed an integral constitutive equation for semiconcentrated fiber suspensions similar to the ones 
obtained by Doi and Edwards [21] and Curtiss and Bird [22] for concentrated solutions of flexible macromolecules. This constitutive equation accounts for fiber-fiber and fiber-liquid interactions and predicts well most of the rheological data previously recorded [23]. In semiconcentrated suspensions, the number density, $n$, of the fibers ranges from $\left(1 / L^{3}\right)<n<\left(1 / D L^{2}\right)$ whereas the volume fraction, $\phi$, varies from $(D / L)^{2}<\phi<(D / L)$ where $L$ and $D$ are the diameter and length of the fiber, respectively. This regime of concentration covers a wide range of reinforced composites because of the considerable fraction of solids present in the system [20].

In this work, we develop the necessary theory and methodology to use these kind of integral constitutive equations, which account for fiber-fiber interactions, with the conservation equations of fluid mechanics, in order to analyze the continuous processing of chopped fibers suspensions. The analysis will predict the flow field and the resulting fiber orientation. Such work with realistic integral constitutive equations for nondilute fiber suspensions has never been undertaken before because: (a) integral constitutive equations for nondilute fiber suspensions appeared very recently [20] and (b) numerical modeling with integral constitutive equation is, in general, not very popular [24].

Numerical modeling with integral constitutive equations is difficult because it demands for each fluid particle and/or each individual fiber an evaluation of past kinematics along a priori unknown streamlines. Papanastasiou et al. [25] developed a streamlined finite-element method in which present kinematics, past kinematics, and streamlines are computed simultaneously for each individual fluid particle (or fiber). The application of this finite-element method to a system of integro-differential equations that arise in flows of viscoelastic liquid is detailed in Papanastasiou et al. [26]. The same method will be used here.

\section{Governing equations}

We focus here on the continuous extrusion of nondilute fiber suspension in a Newtonian solvent (Fig. 1). The extrusion process can be used for the continuous production of composites of several geometric configurations depending on the shape of the die (rod, sheet, tube of different cross-sectional shapes, and annulus). Moreover, the geometry of the flow makes it a good prototype for both confined and free surface and multilayer flows, similar to those taking place in injection and compression molding, and in coextrusion. The geometry of the flow and the relevant boundary conditions are shown in Fig. 1. A fiber suspension emerges from the die at practically zero Reynolds number and rearranges itself into a larger flow conduit-it swells - due to the action of the released normal and shear stresses, and then 


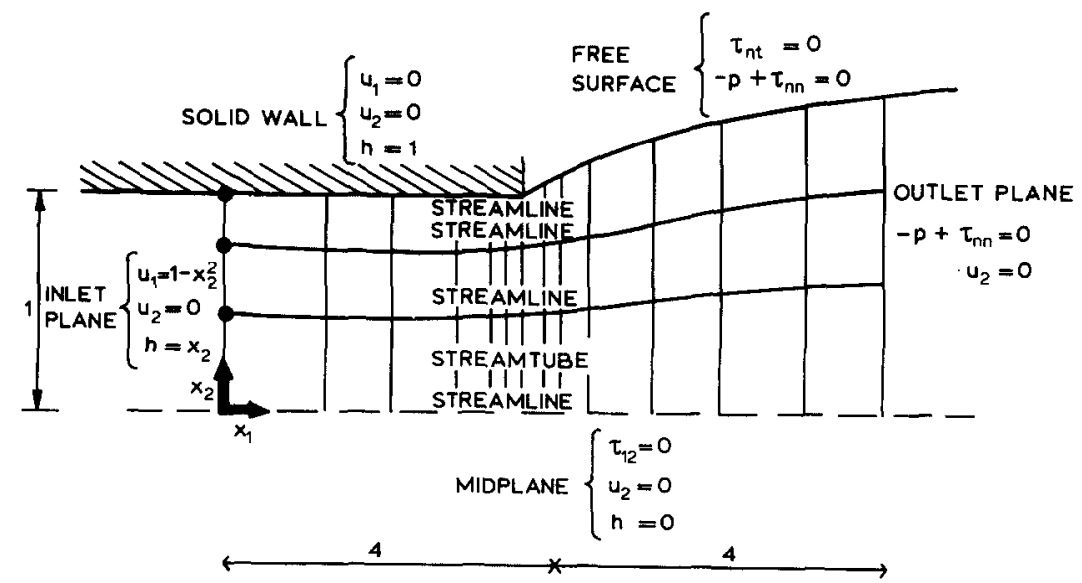

Fig. 1. Flow domain and boundary conditions in a typical extrusion process. In the streamlined finite-element method [25], elements are distributed among selected streamtubes.

assumes a plug-like flow downstream. The lengths upstream and downstream of the die exit were found to be sufficient for a length-independent solution and did not cause any problem as far as the convergence of the Newton iteration was concerned.

In the theory developed Papanastasiou et al. [26], the continuity equation for an incompressible liquid

$\nabla \cdot \boldsymbol{u}(\boldsymbol{x})=0$

the momentum equation for creeping flow

$\nabla \cdot \boldsymbol{T}(\boldsymbol{x})=0$

of liquid that follows an integral constitutive equation

$$
\begin{aligned}
\boldsymbol{T}(\boldsymbol{x})= & -p(\boldsymbol{x}) \boldsymbol{I}+\int_{-\infty}^{x} \sum_{k} \exp \left\{-\frac{1}{\lambda_{k}} \int_{x^{\prime}}^{x} \frac{\mathrm{d} x^{\prime \prime}}{u\left(x^{\prime \prime}\right)}\right\} \\
& \times F\left[\boldsymbol{u}\left(\boldsymbol{x}^{\prime}\right), \boldsymbol{u}(\boldsymbol{x}), \nabla \boldsymbol{u}\left(\boldsymbol{x}^{\prime}\right), \nabla \boldsymbol{u}(\boldsymbol{x})\right] \mathrm{d} x^{\prime},
\end{aligned}
$$

and the kinematic equation

$$
\boldsymbol{n} \cdot \boldsymbol{u}(\boldsymbol{x})=0 \text { with } \boldsymbol{n}=\frac{-u(\partial h / \partial x) i+v \boldsymbol{j}}{\left(1+(\partial h / \partial x)^{2}\right)^{1 / 2}}
$$

are solved simultaneously by Newton iteration which enables the simultaneous evaluation of the streamline position $h$ with the primary unknowns which are the velocities, $u$ and $v$, and the pressure, $p$. The local stress at $x$ in eqn. (2) depends not only on the local pressure $p(x)$ and the velocity 
gradient $\nabla \boldsymbol{u}(\boldsymbol{x})$ alone as in the Newtonian case, but on the past velocities $\boldsymbol{u}\left(\boldsymbol{x}^{\prime}\right)$ and the velocity gradient $\nabla \boldsymbol{u}\left(\boldsymbol{x}^{\prime}\right)$ along the fluid particle's streamline $x^{\prime} \boldsymbol{x}$ as well, which has a projection on the $x$-axis $x^{\prime} x$. The integro-differential eqns. (1)-(4) are easily solvable by the streamlined finite-element method which enables one to calculate simultaneously the present velocity $\boldsymbol{u}(\boldsymbol{x})$ and gradient $\nabla \boldsymbol{u}(\boldsymbol{x})$, past velocities $\boldsymbol{u}\left(\boldsymbol{x}^{\prime}\right)$ and gradients $\nabla \boldsymbol{u}\left(\boldsymbol{x}^{\prime}\right)$, and streamlines $x^{\prime} \boldsymbol{x}$ by using the Newton iteration to solve the resulting set of nonlinear algebraic equations. Conventional finite-element methods are not very convenient for the solution of velocities along a priori unknown streamlines. The streamlined finite-element method is detailed in Refs. 25 and 26.

Quite similar integro-differential systems of equations arise when integral constitutive equations for nondilute fiber suspensions [20]

$$
\begin{aligned}
\boldsymbol{T}(\boldsymbol{x})= & -p(\boldsymbol{x}) \boldsymbol{I}+\eta_{\mathrm{s}}\left[\nabla \boldsymbol{u}(\boldsymbol{x})+\nabla^{T} \boldsymbol{u}(\boldsymbol{x})\right] \\
& \times\left[1+\frac{n L^{3}}{48 \ln \left(2 / n L^{2} D\right)} \int \frac{q q q q}{\left(\boldsymbol{B}^{-1}: q q\right)^{3 / 2}} \mathrm{~d} \boldsymbol{q}\right]
\end{aligned}
$$

are used in place of eqn. (3). In eqn. (5), $\eta_{\mathrm{s}}$ is the viscosity of the solvent, $n$ the number of rigid rod-like fibers per unit volume, $L$ and $D$ the length and the diameter of the fiber. $B^{-1}$ is the Finger tensor and $\boldsymbol{q}$ is the unit vector along the axis of the fiber, i.e. the orientation. The integrand in eqn. (5) can be expressed in terms of a series expansion of the Finger and Cauchy tensors as suggested in [22] and [27]. The Finger and Cauchy tensors, in turn, can be expressed in terms of past and present velocities and gradients along the fiber's streamline as suggested by Adachi [28] and shown in [26]. Equation (5) is detailed in [29].

\section{Finite-element analysis}

Thus eqn. (5) can be transformed into a form which is equivalent to eqn. (3) and can be solved simultaneously with eqns. (1), (2), and (4), by means of finite-element basis functions $\phi^{i}$ and $\psi^{i}$ to determine the flow field (velocities, pressure, and streamlines):

$u=\sum_{1}^{9} u_{i} \phi_{i}, \quad v=\sum_{1}^{9} v_{i} \phi_{i}, \quad p=\sum_{1}^{4} p_{i} \psi_{i}, \quad h=\sum_{1}^{3} h_{i} \phi^{i}(y=h)$.

The expansions (6) are substituted in eqns. (1), (2), and (4), the resulting equations are weighted integrally by each of the basis functions, the divergence theorem is invoked to project the boundary terms, and the boundary terms are replaced by the known tractions or else the entire weighted residuals are replaced by essential boundary conditions there. These 


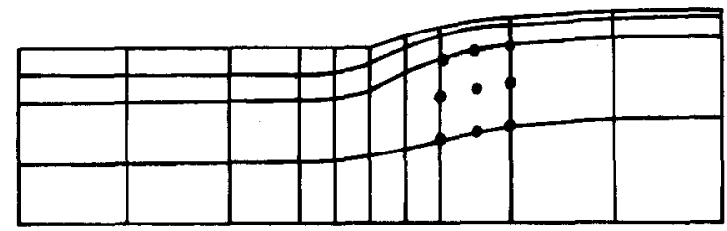

Fig. 2. The streamlined finite-element tessellation used in this work. The location of the streamlines and, thus, the mesh are updated at each Newton iteration. The tessellation extends four half-widths upstream and four downstream from the die-exit.

boundary conditions are shown in Fig. 1. This results in a system of nonlinear algebraic equation which is solved by Newton iteration:

$\boldsymbol{J}\left(\boldsymbol{p}^{(n+1)}-\boldsymbol{p}^{(n)}\right)=-\boldsymbol{R}\left(\boldsymbol{p}^{(n)}\right)$.

Here $\boldsymbol{R}$ is the vector of the weighted residuals, $\boldsymbol{p}$ is the vector of the unknowns, $\boldsymbol{p}=\left\{u_{1}, v_{1}, p_{1}, h_{1} \ldots U_{N}, V_{N}, P_{N}, h_{N}\right\}$, and $J$ is the Jacobian of $\boldsymbol{R}$ with respect to the nodal unknowns $\boldsymbol{p}$.

The tessellation of the flow field in quadrilateral elements is shown in Fig. 2. The finite elements are distributed among four streamtubes. The boundaries of each individual streamtube are made up by the lateral sides of the contained elements. The two velocity components are expanded in terms of the biquadratic basis functions, the pressure in terms of the bilinear functions, and the streamlines in terms of the quadratic basis functions. The solution, at a given concentration level, serves as the initial estimate of the solution at a higher concentration level (zero-order continuation) required by the Newton iteration. The streamlines, including the free surface, are
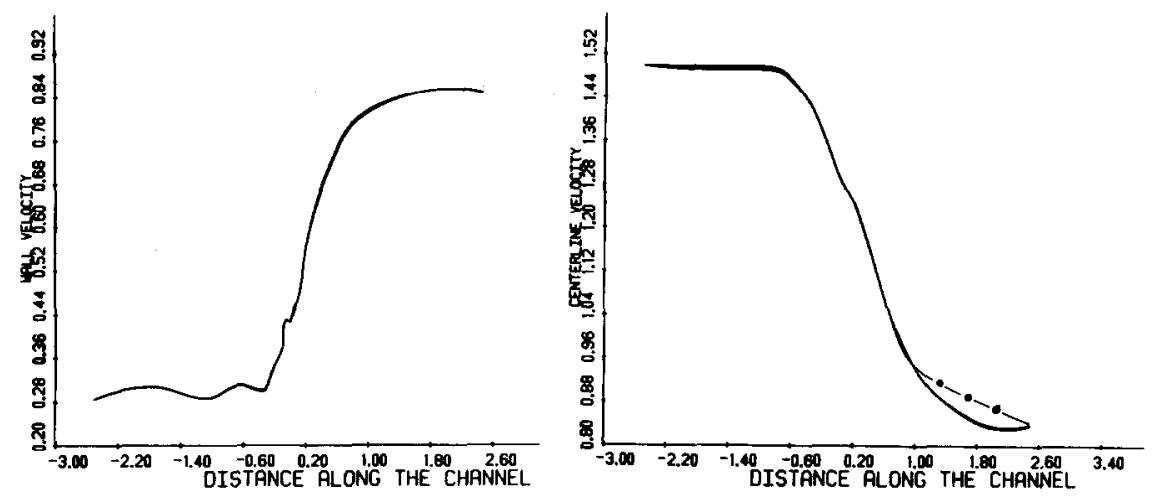

Fig. 3. Predicted velocity profiles of suspension of fiber of aspect ration $L / D=10$ at three levels of concentration: - . . . - dilute of fiber volume fraction $\phi=0.01,-C-$ nondilute of $\phi=0.05$ and $\cdot-\cdot-\cdot$ semiconcentrated of $\phi=0.1$. 
updated and so is the tessellation at each Newton iteration. A finer tessellation is necessary near the static contact line to resolve the local large gradient. The tessellation shown was found sufficient to produce mesh-independent solutions.

\section{Fiber orientation}

The slender fiber orientation $q(x)$ at location $x$ is determined from its initial orientation $\boldsymbol{q}_{0}\left(x_{0}\right)$ at the inlet and the deformation gradient $\boldsymbol{F}_{\mathrm{x}_{0}}(x)$ between $x$ and $x_{0}$ which is readily available from the solution of the system of eqns. (1), (2), (4), and (5):

$\boldsymbol{q}=\left(\boldsymbol{F}_{x_{0}}(x) \cdot \boldsymbol{q}_{0}\right) / M$.

The denominator $M$ is the magnitude of the numerator and dividing by it forces $q$ to remain a unit vector.

In dilute fiber suspensions the orientation can be deduced by tracking each individual fiber rotating about its center as it translates along its streamlines with the bulk velocity. Whereas this is fairly accurate for dilute suspensions, and away from solid walls, the orientation function distribution is better suited for a nondilute suspension. Dinh and Armstrong [20] produced an analytic expression for the orientation function distribution $\psi(q, t)$ used in the derivation of the constitutive eqn. (5)

$\psi(\boldsymbol{q}, t)=\frac{1}{4 \pi}\left[\boldsymbol{B}_{t_{0}}^{-1}(t): q q\right]^{-3 / 2}$,

where $\boldsymbol{q}$ is the orientation vector and $\boldsymbol{B}_{0}^{-1}(t)$ the deformation between a reference entering time $t_{0}$ and a later time $t$. The initial conditions for eqn. (9) are

$\boldsymbol{B}_{t_{0}}^{-1}\left(t_{0}\right)=\boldsymbol{I}, \quad \psi\left(\boldsymbol{q}, t_{0}\right)=\psi_{0}=1 / 4 \pi$,

where $I$ is the unitary tensor and $\psi_{0}$ is an initially random fiber distribution at entering time $t_{0}$. In the results reported here, the fiber orientation was calculated by means of eqns. (9) and (10).

\section{Results and discussion}

The Newton iteration converged quadratically to the reported results in four to five iteration steps within maximum error $10^{-4}$. The results for a Newtonian liquid were reproduced exactly in the limiting case of zero concentration. Unlike the numerical solution for a viscoelastic liquid, which is limited to low elasticity, the solution here exists at any concentration we examined as shown by Fig. 4-12. 

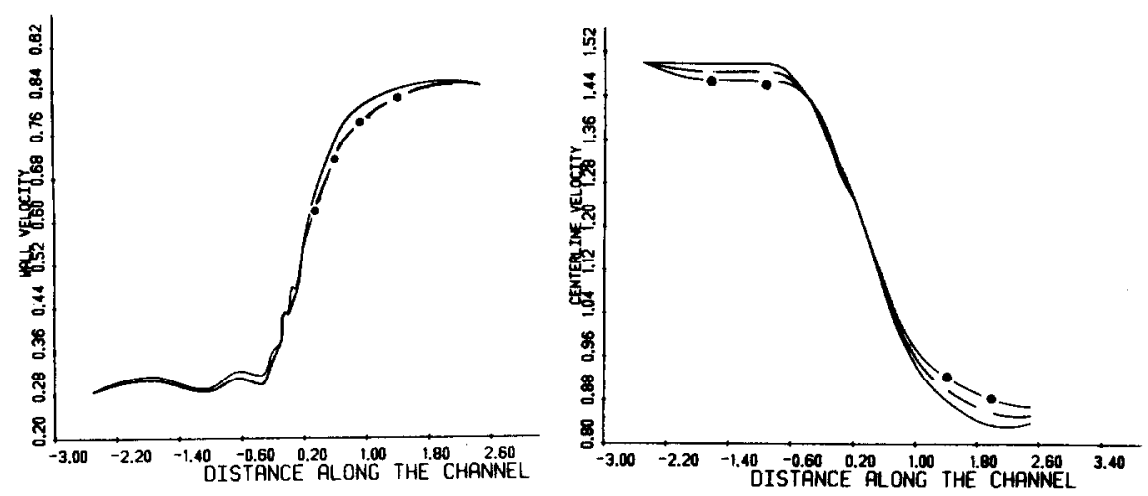

Fig. 4. Predicted velocity profiles of suspension of fiber of aspect ratio $L / D=100$ at three levels of concentration: - . - . - - dilute of fiber volume fraction $\phi=0.001,-\ldots$ nondilute of $\phi=0.005$ and $\cdot-\cdot \cdot \cdot$ semiconcentrated of $\phi=0.01$.
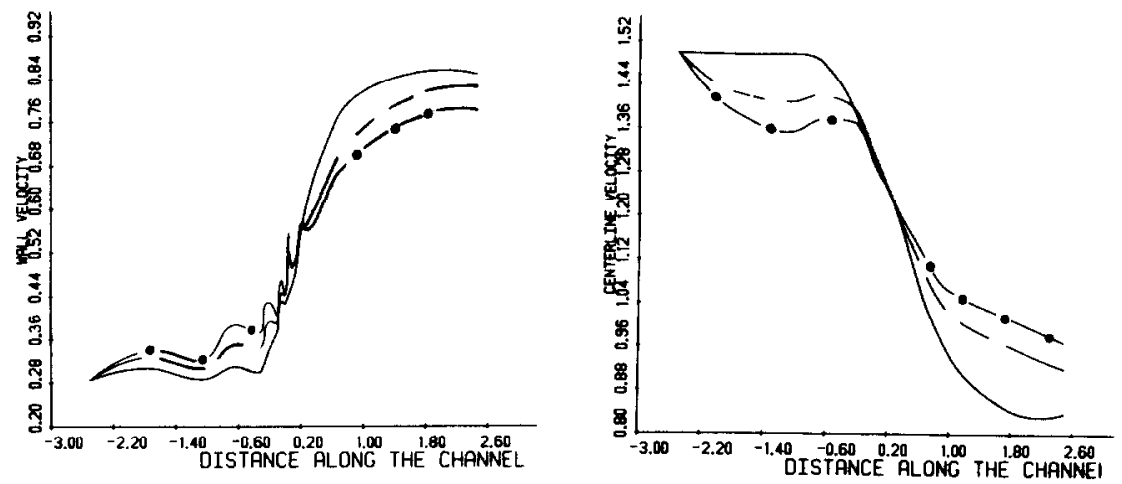

Fig. 5. Predicted velocity profiles of suspension of fiber of aspect ration $L / D=100$ at three levels of concentration: -..... dilute of fiber volume fraction $\phi=0.000001, \ldots$ nondilute of $\phi=0.00005$ and $\cdot-\cdot-\cdot$ semiconcentrated of $\phi=0.001$.
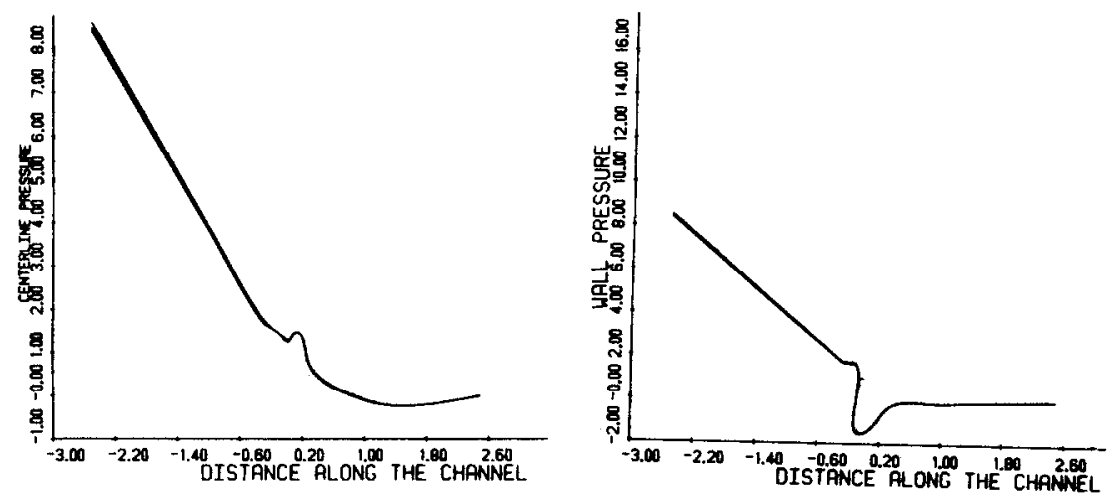

Fig. 6. Predicted pressure distribution in suspension of fiber of aspect ratio $L / D=10$ at three levels of concentration: - - - - - dilute of fiber volume fraction $\phi=0.01,-\ldots$ nondilute of $\phi=0.05$ and $\cdot-\cdot \cdot$ semiconcentrated of $\phi=0.1$. 

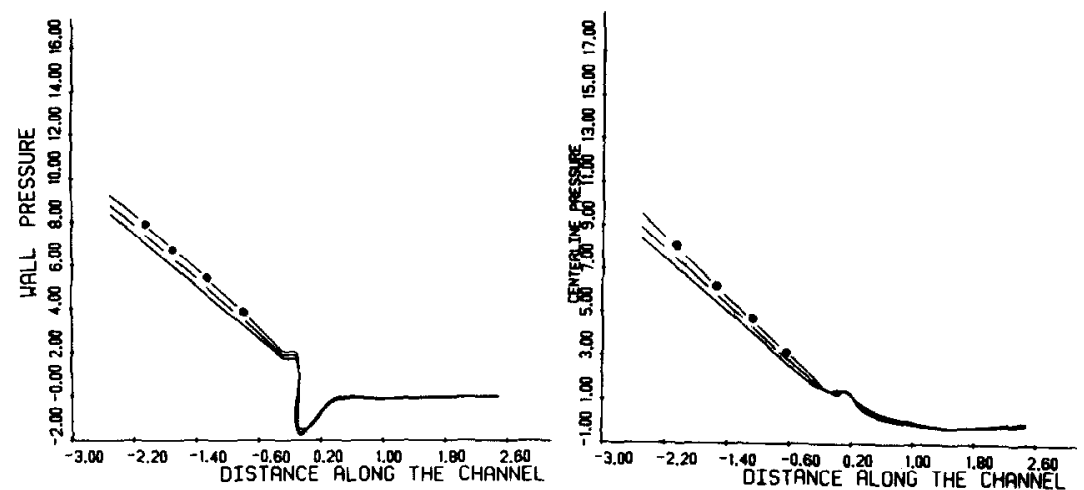

Fig. 7. Predicted pressure distribution in suspension of fiber of aspect ratio $L / D=100$ at three concentration levels: $\ldots \ldots$. dilute of fiber volume fraction $\phi=0.0001, \ldots$ nondilute of $\phi=0.005$ and $\cdot \cdots \cdot \cdot$ semiconcentrated of $\phi=0.01$.
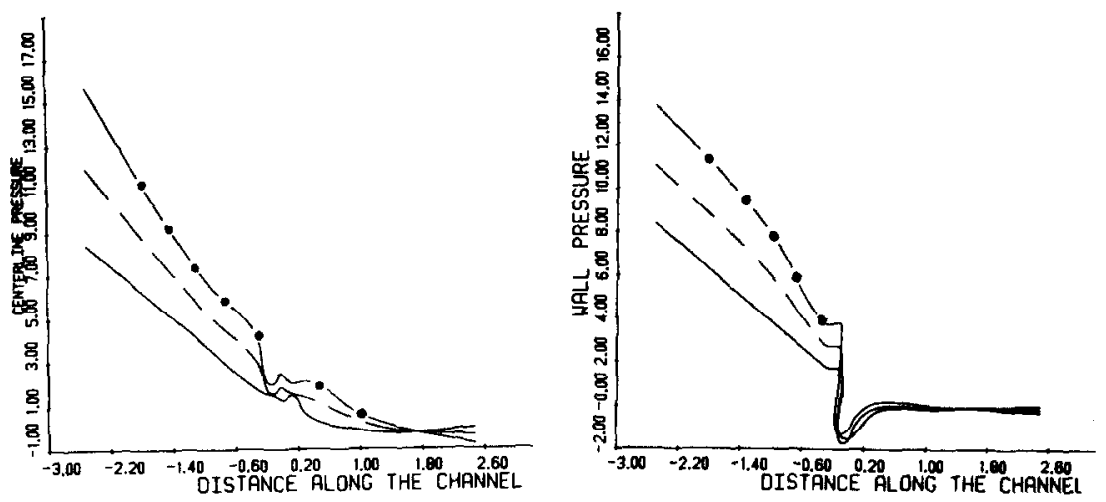

Fig. 8. Predicted pressure distribution in suspension of fiber of aspect ratio $L / D=1000$ at three concentration levels: - . . . . dilute of fiber volume fraction $\phi=0.000001$, $-\ldots$ nondilute of $\phi=0.00005$ and $\cdot-\cdot-\cdot$ semiconcentrated of $\phi=0.001$.

The three levels of concentration mentioned in the reported results are defined as follows: dilute when $n=1 / L^{3}$ where $n$ is the number of fibers per unit volume and $L, D$ the length and diameter of the fiber, respectively, or equivalently when $\phi=(D / L)^{2}$, where $\phi$ is the volume fraction of the fibers; nondilute when $n=0.5\left(1 / L^{3}+1 / D L^{2}\right)$ or when $\phi=0.5(D / L)^{2}+$ $(D / L)$ ); and semiconcentrated when $n=1 / D L$ or $\phi=D / L$. At higher concentrations, solid-like theories are required. Nevertheless, the regions covered here contain enough fibers per unit volume to be representatives of some composite materials [20]. 

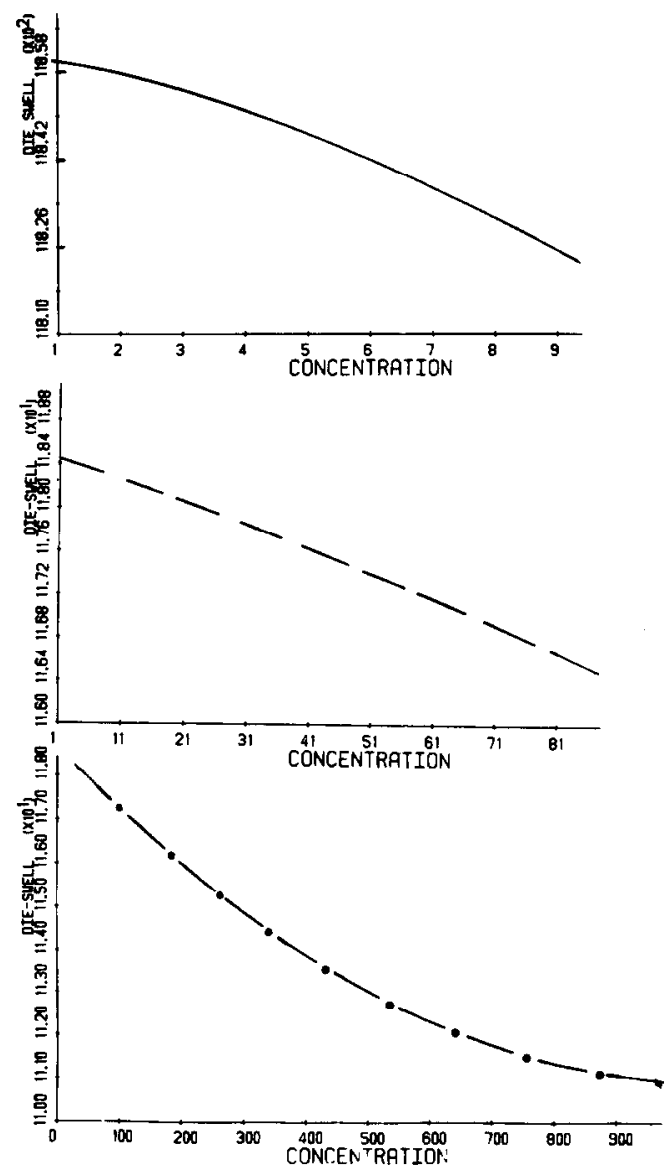

Fig. 9. Predicted die-swell of suspension of fiber of aspect ratio: $--\cdots-L / D=10,---$ $L / D=100$ and $\cdot-\cdot-\cdot L / D=1000$ at several fiber concentration (fibers per unit volume) levels.

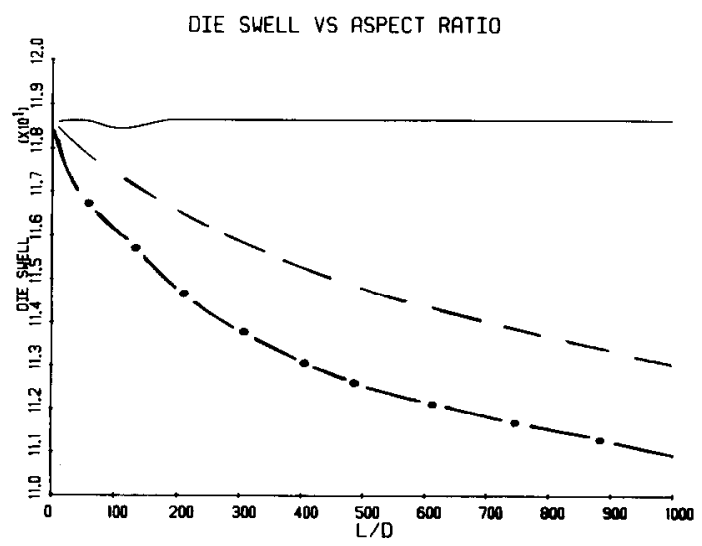

Fig. 10. Predicted die-swell of suspension of fiber of several aspect ratio: - - . - dilute of fiber volume fraction $\phi=(D / L)^{2}--$ nondilute of $\phi=0.5\left[(D / L)^{2}+(D / L)\right]$ and ...-. semiconcentrated of $\phi=(D / L)$. 
- $\frac{L}{D}=1, n=1, \theta=90^{\circ}$ (SPHEROID)

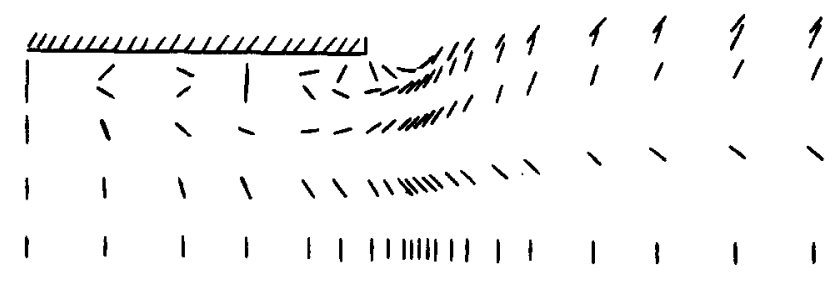

- $\frac{L}{D}=1, n=1, \theta=0^{\circ}$ (SPHEROID)

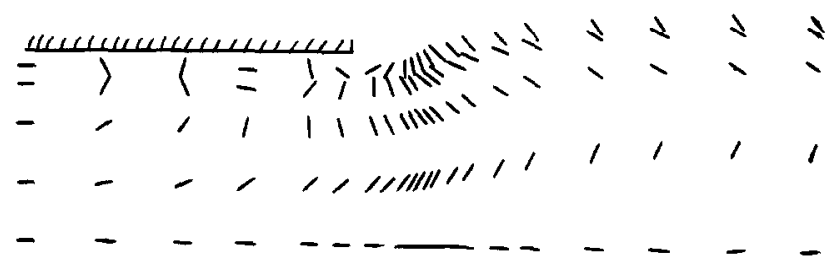

Fig. 11. Predicted orientation in a suspension of a spherical fiber entering perpendicularly (top) and parallel (bottom) to the flow direction.
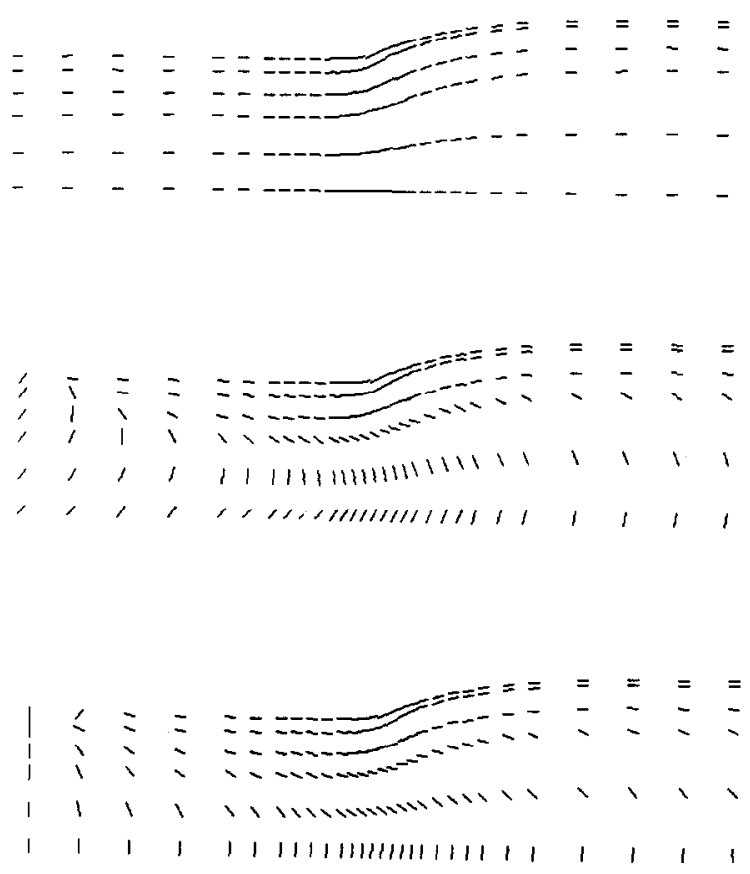

Fig. 12. Predicted orientation in a suspension of slender fiber of $L / D=100$ entering parallel (top), at $45^{\circ}$ angle (middle), and perpendicularly (bottom) to the flow direction. 


\subsection{Processing conditions}

Figure 3 shows the predicted velocity along the wall (distance 0.1 diameters from the wall) and along the centerline for dilute (i.e. limits of zero concentration), nondilute, and semiconcentrated (i.e. upper limit of nondilute region) rod-like fiber suspension of short fiber. Figures 4 and 5 show the corresponding results for suspensions of long and slender fibers, respectively. Suspensions of short fiber $(L / D=10)$ behave identically at the three levels of concentration which correspond to the volume fraction of fibers of $0.01,0.05$, and 0.1 , respectively. For the long fiber, the volume fraction of the fiber is 0.0001 for dilute, 0.005 for nondilute and 0.01 for semiconcentrated, suspensions. Although of low fiber volume fraction, these suspensions exhibit slightly different velocity profiles both along the wall and the free surface and along the midplane streamlines. The differences grow large for a slender fiber of aspect ratio $L / D=1000$ as Fig. 5 illustrates. The three levels of fiber volume fraction are $0.000001,0.00005$, and 0.001 , respectively.

The results of Figs. 3-5 indicate that the deviations of fiber suspensions from the plain solvent behavior depend strongly on the fiber's shape anisotropy and weakly on the fiber concentration even at very low fiber concentrations. The behavior of the fiber suspension is qualitatively different from the behavior of a viscoelastic liquid where the wall velocity is increased inside the channel and decreased under the free surface relative to the solvent velocity. This qualitative difference exists for the centerline velocity too.

Figures 6-8 show the pressure along the solid wall and the centerline for short, long, and slender fibers, respectively. Suspensions of short fiber behave similarly to the plain solvent at all levels of concentration. Suspensions of long fiber behave slightly different from the plain solvent. This difference grows large for suspensions of slender fiber which exhibit a large pressure drop and, thus, volume fraction-thickening behavior. Unlike viscoelastic liquids, all the pressure curves here are reasonably smooth, which may indicate a solvent dominated stress perhaps due to fibers aligned parallel to the wall. Finally, it is worth mentioning here that the Newtonian behavior is predicted well in the dilute limit of all three aspect ratios. This is important given the lack of experimental data or results of other analyses to compare with the results of this work.

The predicted die-swell for fiber aspect ratios $L / D=1 / 0.1,1 / 0.01$ and $1 / 0.001$ is shown in Fig. 9 as a function of concentration from dilute to semiconcentrated suspensions. The Newtonian behavior is predicted well in the dilute limit. The die swell decreases with fiber concentration and with the aspect ratio of the fiber. This is better demonstrated in Fig. 10. The die-swell for dilute suspension is almost identical to the Newtonian value, 
1.185 , at any fiber aspect ratio; for semiconcentrated and concentrated suspensions, the die-swell decreases drastically with the aspect ratio. The die-swell predictions suggest a shear-thinning behavior intensified by large aspect ratio.

\subsection{Fiber orientation}

Figure 11 shows the predicted orientation for a sphere $(L / D=1)$ entering at 0 and 90 degrees with respect to the flow direction. In reality, this means the orientation of the two "marked" at the entrance, mutually perpendicular axes of the spheroid. As is expected, the sphere turns under the vorticity in such a way that the two axes remain mutually perpendicular

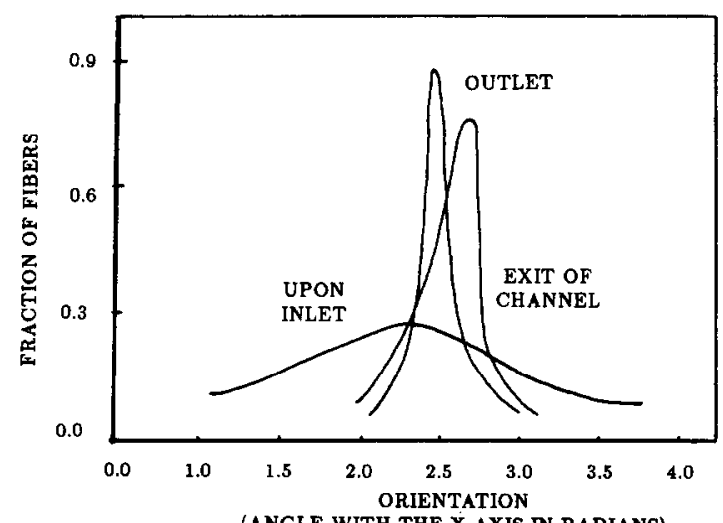

(ANGLE WITH THE X-AXIS IN RADIANS)

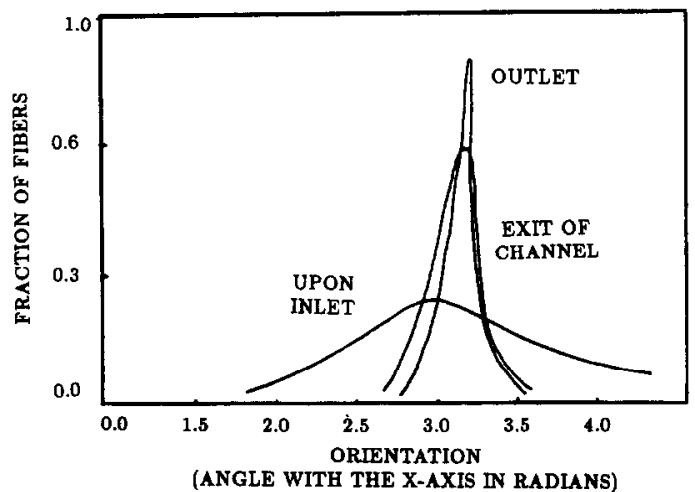

Fig. 13. Orientation function distribution along two selected streamlines of Fig. 12 by means of eqn. (9) for an initial (entering) random orientation. Top: Along streamline next to the midplane of symmetry. Bottom: Along streamline next to the midplane of symmetry. Bottom: Along streamline next to the solid wall and the free surface. 
at all locations downstream. This is an indication that the time integration along the streamline, which we used to solve eqn. (9) from the flow field, is stable and accurate.

Figure 12 shows the predicted orientation for a single slender fiber $(L / D=100)$. When the fiber enters parallel to the flow it remains aligned with the streamline at all downstream locations. When the fiber enters perpendicularly to the flow it is subject to a continuous rotation which finally aligns the fiber to its streamline. However, the fiber is subject to a solid translation without rotation as soon as it enters the extensional flow region outside the die. Thus, if the fiber enters the extensional flow not aligned with the streamline it will never become so downstream. The perpendicular orientation along the midplane streamline is unstable and, once disturbed by flow or vibration, it never re-establishes itself.

The evolution of the orientation function distribution starting from an random orientation entering along two selected streamlines of Fig. 12 is shown in Fig. 13 at three locations: immediately upon entry, at the channel exit, and far downstream near the outlet. The orientation function distribution was computed by solving eqn. (9) along the streamline with the initial (entering) condition given by eqn. (10). It is obvious that the most preferred orientation of a slender fiber is one parallel to the streamlines and the possibility of this orientation increases with downstream distance.

\subsection{Implications for viscoelastic calculations}

A comparison of the numerical stability of the computations of this work to those for the die-swell flow of a viscoelastic liquid modelled by integral constitutive equation appears inevitable: the equations for fiber suspension, eqn. (5), and for a viscoelastic liquid, eqn. (6), differ only by the exponential time series included in the memory function of the latter. In fact, the predictions of the two equations in many simple rheological experiments are qualitatively similar $[20,23]$. It is well known that numerical solutions for a viscoelastic liquid are limited to low elasticity. One of the possible causes suggested for this failure is an inaccurate calculation of strains along streamlines [30]. Although the strains calculated here are identical to those required in the flow of a viscoelastic liquid, the solution here exists at any concentration limit where eqn. (5) holds and predicts substantial viscoelastic behavior [23]. Thus, it appears that the primary cause (among others) of numerical difficulties in viscoelastic calculations is the exponential time-dependent series rather than the strain-dependent part of the memory function of the constitutive equation. This, in turn, may suggest constitutive equations with a non-exponential relaxation modulus for a viscoelastic liquid; for example, of the form proposed by Larson [31] recently. 


\section{Conclusion}

The isothermal extrusion of a nondilute fiber suspension, as a first step towards the continuous production of composite materials, has been analysed. The analysis has demonstrated the possibility for process control to achieve specified orientation patterns and, thus, composites of desired properties. The fiber orientation can, in principle, be controlled by a knowledge of the velocity and the temperature fields. These fields are predicted by the application of the preceding analysis to nonisothermal extrusion.

From the practical point of view, the preceding analysis showed that, in principle, a certain class of composites can be processed in a continuous fashion with the possibility of predicting and controlling fiber orientation. The constitutive equation developed recently by Dinh and Armstrong [20] and the theory developed recently by Papanastasiou et al. [26] for liquids with memory have made this analysis possible. At present, the analysis is limited to Newtonian matrices which approximate well epoxy composites. The analysis is also limited to nondilute semiconcentrated suspensions which can represent well many commercial composites. These limitations are entirely due to limitations in the applicability of the constitutive equation.

From a theoretical point of view, we have demonstrated an effective computer-aided analysis of a certain class of integro-differential systems similar to the ones arising in processing viscoelastic liquids that follow integral constitutive equations. The differences between a nondilute suspension and a viscoelastic liquid regarding their flow behavior and the stability of the numerical algorithms may suggest another approach to the general viscoelastic problem. Such research is in progress.

The analysis can only be viewed as a first step towards understanding and predicting the behavior of nondilute fiber suspensions that lead to the production of composite materials of significant fiber volume fraction. Further investigation is in progress which includes a more efficient use of the orientation distribution function for better prediction of fiber orientation patterns, the control of the solidification front in a nonisothermal flow field, and experimentation with epoxy-fiber glass systems for rheological characterization and frozen-in orientation measurement.

\section{Acknowledgements}

This research was supported by grants in aid by the National Science Foundation CBT-8504607/Program of Process and Reaction Engineering. 


\section{References}

1 M.J. Owen, D.H. Thomas and M.S. Found, British Plastics Federation, Proc. Reinforced Plastics Congr. Paper 18 (1978).

2 M.W. Darlington and P.L. McGinley, J. Mater. Sci., 10 (1975) 906.

3 H. Goldsmith and S.G. Mason, in Eirach (Ed.) Rheology: Theory and Applications, Academic Press, 1967, p. 85.

4 F.J. Lockett, Plast. Rubber: Proc. 6 (1981) 85.

5 L.G. Leal, Ann. Rev. Fluid Mech., 12 (1980) 435.

6 R.C. Givler, M.J. Crochet and R.B. Pipes, J. Compos. Mater., 14 (1983) 330.

7 R.C. Givler, Numerical techniques for the prediction of flow induced orientation, Ph.D. Thesis, Univ. Delaware, 1983.

8 L.A. Goettler, Proc. Ann. Conf. Reinforced Plast./Comp. Inst., Soc. Plast. Ind., 25, Sect. 14-A, (1970) 1.

9 A. Metzner, J. Rheol., 29 (1986) 739.

10 W.R. Blakeney, J. Colloid Interface Sci., 22 (1966) 324.

11 E.L. Rosinger, R.T. Woodhams and C.E. Chaffey, Trans. Soc. Rheol., 18 (1974) 453.

12 M. Horie and K.L. Pinder, J. Chem. Eng., 57 (1979) 125.

13 R.O. Maschmeyer and C.T. Hill, Trans. Soc. Rheol., 21 (1977) 183, 195.

14 C.L. Tucker III, Reaction injection molding of reinforced polymer parts, Ph.D. Thesis, M.I.T.

15 J-N. Miles, N.K. Murty and G.F. Molden, Polym. Eng. Sci., 21 (1981) 1171.

16 T.E. Kizior and F.A. Syere, Trans. Soc. Rheol., 18 (1974) 271.

17 C.B. Weinberger and J.D. Goddard, Int. J. Multiphase Flow, 1 (1974) 465.

18 J. Mewis and A.B. Metzner, J. Fluid Mech., 62 (1974) 593.

19 M. Khagram, R.K. Gupta and T. Sridhar, J. Rheol., 29 (1985) 191.

20 S.M. Dinh and R.C. Armstrong, J. Rheol., 28 (1984) 207.

21 M. Doi and S.F. Edwards, J. Chem. Soc. Farad. Trans., 74 (1978, 1979) 510, 911.

22 C.F. Curtiss and R.B. Bird, J. Chem. Phys., 74 (1981) 2016, 2026.

23 M.A. Bibbo, S.M. Dinh and R.C. Armstrong, J. Rheol., 29 (1985) 905.

24 M.J. Crochet and K. Walters, Ann. Rev. Fluid Mech., 15 (1983) 241.

25 A.C. Papanastasiou, C.W. Macosko and L.E. Scriven, Streamlined finite element and transit times, in Gallagher, Oden, Carey, and Zrenkiewicz (Eds.), Finite Element Fluids, John Wiley and Sons, 1985.

26 A.C. Papanastasiou, L.E. Scriven and C.W. Macosko, J. Non-Newtonian Fluid Mech., 22 (1987) 271.

27 P.K. Currie, J. Non-Newtonian Fluid Mech., 11 (1982) 53.

28 K. Adachi, Rheol. Acta., 22 (1983) 326.

29 S.M. Dinh, On the rheology of concentrated fiber suspensions, Ph.D. Thesis, MIT, 1981.

30 B. Caswell and K. Viriyayuthakorn, J. Non-Newtonian Fluid Mech., 12 (1983) 13.

31 R.G. Larson, Rheol. Acta, 24 (1985) 327. 